\title{
Recycling Construction Waste Material with the Use of AR
}

\author{
Caitlyn Parry ${ }^{1(\otimes)}$ and Sean Guy ${ }^{2}$ \\ 1 RMIT University, Melbourne, Australia \\ Caitlyn.parryarmit.edu.au \\ ${ }^{2}$ Fologram, Melbourne, Australia \\ Seanefologram.com
}

\begin{abstract}
This paper aims to present a methodology for reusing and recycling scrap timber from building sites using augmented reality and flexible digital models. The project we present describes a process that enables existing material to be reused and repurposed such that the designed model is updated by the digital inventory of digitised offcuts/waste elements.
\end{abstract}

Keywords: Material reuse - Material recycling - Augmented reality fabrication . Digitization of physical parts

\section{Introduction}

The construction industry is one of the greatest contributors to global pollution via both the carbon emissions from the production of construction material and its direct contribution to landfill. From 1900 to 2010, the amount of materials accumulated in buildings and infrastructure across the world increased 23 -fold (Krausmann et al. 2017). Annually, the construction and demolition industry produce over 20 million tonnes of waste. The waste material from these industries is mainly constituted from timber, metal, concrete, bricks, rock and soil (Shooshtarian 2019).

The development of the digital twin pertaining to smart cities has enabled the capacity to model, simulate and observe over time, the complex interactions of the city and technology (Mohammadi et al. 2017) however it has yet to harness any future potential inherent in the embodied material resources of the city. The Smart City is an advanced piece of infrastructure, and until every built form is digitised specifically as an inventory of materials, down to the lengths of timber, there remains an opportunity to work with the collection of site offcuts and landfill bound materials.

\section{Aims}

This paper seeks to present a methodology for using mixed reality in the documentation, fabrication and construction using waste building material. There is a gap in the current construction industry which, until each building has shifted to also exist as it's digital twin 
means that buildings are demolished with crude attempts to recycle and subsequently no real scalable or economically viable pathways for reuse. By using mixed reality fabrication methodologies for the digitization and assembly of mass-customized scrap timber pieces, we aim to demonstrate how scrap material can be effectively re-used in construction without the need for advanced material manipulation and wastage.

This paper presents two approaches for the re-use of waste material.

The first project categorizes timber scrap material pieces of unique lengths but with similar cross section profiles that are used as a best fit for a designed timber aggregation. This research is explored in a workshop for the AA Visiting School Melbourne 2019 \& Design Studio at RMIT University 2019 with the intention to reduce the need to machine odd shaped offcut materials through the construction of a timber assembly of more than 600 uniquely shaped pieces.

The second project, initially set up as a workshop for the 2020 Melbourne Design Week, extends the logic further to develop a workflow that eliminates any material wastage. It also expands the usability of differentially sized timber from the similar elements used in project 1. A dynamic digital model is updated in response to the input of each timber part's dimensions. This digital model updates and adapts to the kit of parts fed into it. In addition to developing a method for a developable digital model, a process for real like geo located inventory of the scanned directs assembly workers to the location of each part to be retrieved. 'Digital twin' models are typically developed as a digitised model first, or computed from large data sets. The aim of this paper is to present a simpler method for digitisation and digital model making.

\section{Method}

Both of the following projects and methodologies discussed here make use of the Fologram plugin for Grasshopper (Jahn et al. 2020) which enables the augmented visualisation of geometry via either smart phones or AR headsets.

\subsection{Method 1 I Mass Customisation and Working to a Fixed Digital Model}

\subsubsection{Recycled Offcut Timber Scrap Material}

This project developed a methodology of working with offcuts of scrap timber that were machined to fit to a relatively fixed digital model. As discussed by Jahn et al., the digital model acts as a template to guide material location. The timber offcuts were sourced from a local furniture company, with no repeatability in their length, width or height from piece to piece. A variety of breeds of timber were used in the project, ranging from Tasmanian Oak, Ash and Beech. The timber pieces were categorized into groups based on self-similar cross section profiles, but with significantly varying lengths measuring as short as $50 \mathrm{~mm}$ and as long as $2000 \mathrm{~mm}$. Profiles with cross sections between 20$30 \mathrm{~mm}$ in both dimensions were selected for mark-up in augmented reality. These cross section sizes were chosen for a visual density of the timber in the design, as well as for self-similar surface areas for face on face joints (Fig. 1). 


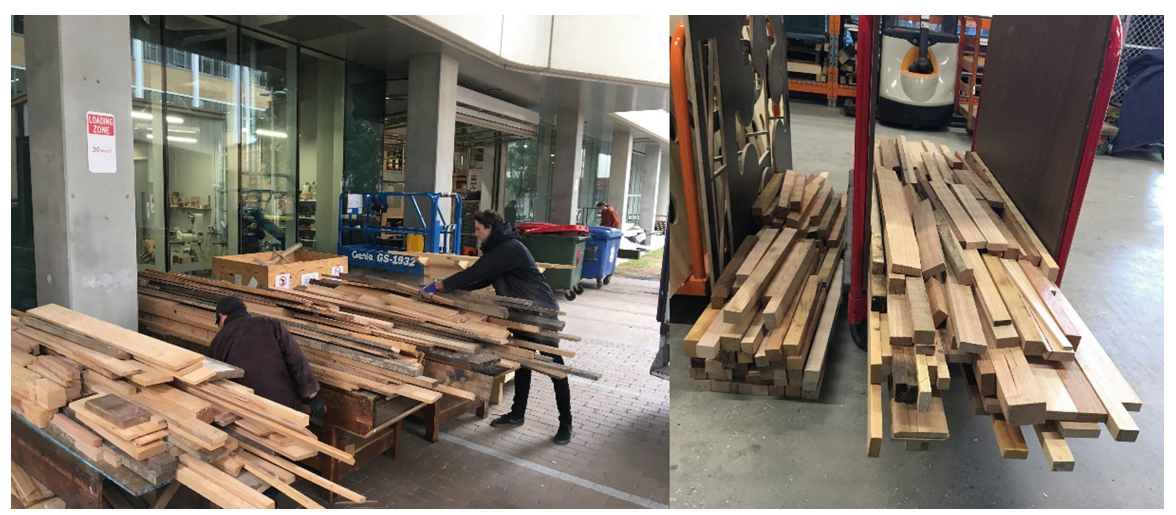

Fig. 1. A variety of timber offcuts categorized by their cross section profiles

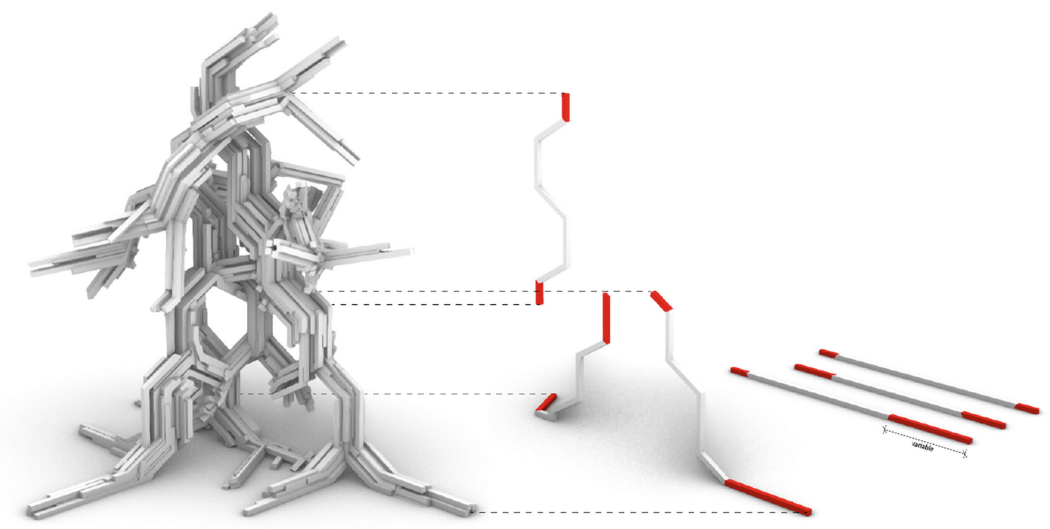

Fig. 2. L-System Field aggregation with dynamically nested assemblies for custom timber lengths

\subsection{Mass Customized Aggregation Geometry}

A simple parametric model was created from a rule based L-System aggregation. A customized field system was simulated using the L-System as a guide to create variation in the parts of the aggregation. These field lines were broken down into smaller discrete elements to approximate the locations of the offcut scrap timber. These design decisions were made to transform a typically standardized design language of an L-System into an aggregation of mass customized adaptable parts. Every angle in the aggregation was standardized to $30^{\circ}$ to reduce material wastage in the cutting process. A standardized angle meant that each piece could be dynamically nested to one another, and then fit to the odd shaped timber sizes. Because these tessellations would never fit the timber lengths perfectly, a customizable 'End piece' (shown in red in Fig. 2) was designed within the aggregation to be variable in length to eliminate timber offcut wastage in the marking of the timber lengths. These end pieces affected the final outcome, so rather 
than the final construction representing a physical twin of the digital, the process led to an adaptable outcome that changed during the fabrication process.

\subsection{Holographic Part Nesting}

Rather than writing a complex algorithm to nest the timber pieces in each odd length, assistants to the project were able to use the Microsoft HoloLens to project holographic cutting templates and dynamically fit pieces inside each offcut timber part. Assistants were able to live stream each part from the aggregation as an overlay on each timber offcut through Fologram. The holographic overlay provided a wireframe of each part, the part number, information about the location of each cutting angle, the length of each edge on the part, and a sanity check for each angle to ensure the digital model adhered to a standardized angle of $30^{\circ}$. Assistants to the project were able to accurately measure and mark the timber scrap lengths against this holographic guide of each part in the digital model without the use of any drawings (see Fig. 3). Once a part had been marked, the next part would be aligned at the cutting location of the previous mark to tessellate and nest the pieces together. Participants worked from the inside (middle) of each offcut scrap and outward, so the end pieces could be variable in length. This process allowed for a dynamic nesting of parts, so rather than relying on a complex nesting algorithm, assistants were able to use their own intuition based on the holographic guides in front of them to decide which pieces would best fit along each timber length.

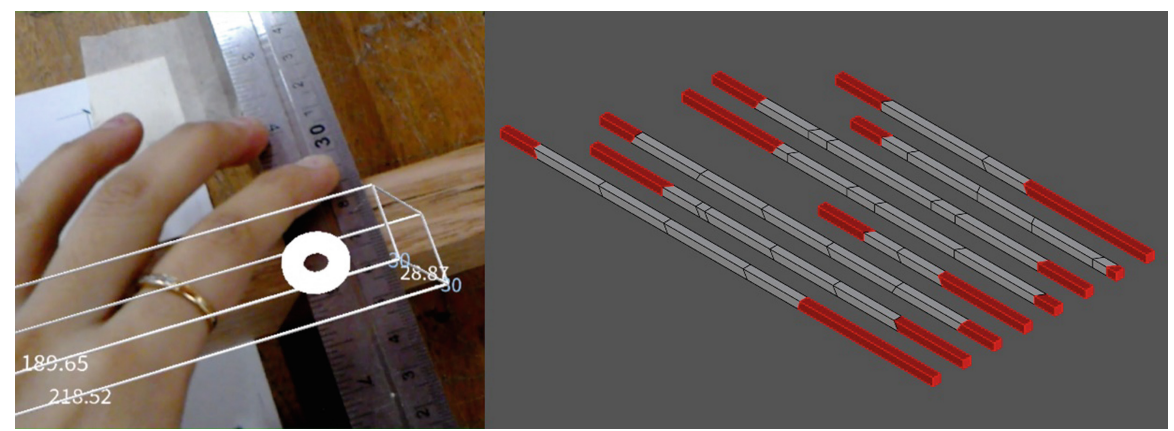

Fig. 3. Parts were dynamically nested within offcut scraps using a holographic guide and user intuition

\subsection{Mixed Reality Interface}

An interactive mixed reality interface was designed by assistants to change marking instructions for each timber offcut piece directly inside the Microsoft HoloLens (see Fig. 4). This meant changes to the holograms being displayed could be adjusted without the need for direct interaction with the Rhino \& Grasshopper interface. The interface contained a series of buttons that allowed assistants to cycle through each piece in the aggregation, nest the hologram to their timber length and rotate each holographic guide to 
align with previous pieces to mark each part end for cutting. The interface also included different shading options for viewing the hologram. Wireframe holograms were found to be more accurate to use for markings than shaded holograms, as the edges were clearer and easier to align. As each piece was overlayed on each offcut scrap, assistants could toggle on an option that would visualize where a particular piece was located in the overall aggregation.

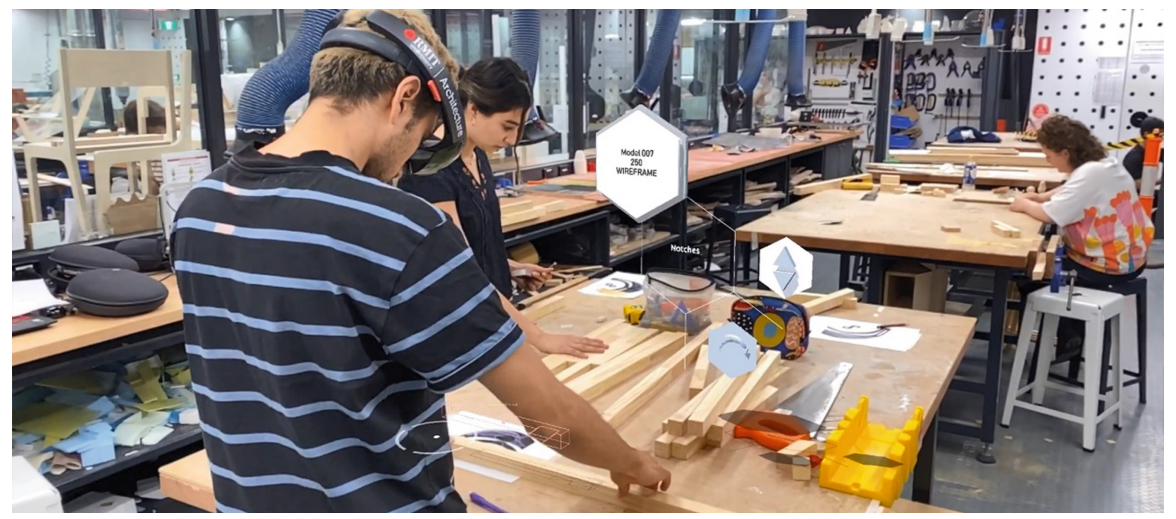

Fig. 4. A holographic interface was used to update aggregation parts for a dynamic nesting in the offcut scraps

\subsection{Jointing}

Once an offcut piece had been marked and nested, the piece was cut on a drop saw set to a standardized angle of $30^{\circ}$. The standardization of the joint meant each piece could be cut quickly and efficiently. Approximately $2.5 \mathrm{~mm}$ length was lost in each part during the cutting process, so this length was adjusted for in the holographic overlays in the marking process to allow for accurate fabrication of each part. Two simple methods were used as joint systems in the assembly: a standardized domino joint system commonly found in furniture making and nail guns into planar facing pieces. A specialized jig (see Fig. 5) was setup alongside a domino machine to accurately and rapidly fabricate the joints for each part in the aggregation.

\subsection{Fabrication and Fixing Methods}

The overall aggregation was broken down into a series of chunk studies to enable multiple users to assemble the final timber aggregation simultaneously. Assistants made use of their interactive interface to control the number of parts they saw in each chunk at one time. This interface also contained information about the part numbers, so users could clearly see which part belonged in which location, and which parts it was connected to. Once each chunk was assembled, assistants used an overall holographic model to piece together each part in the aggregation. Offcut scrap pieces not used in the marking and 


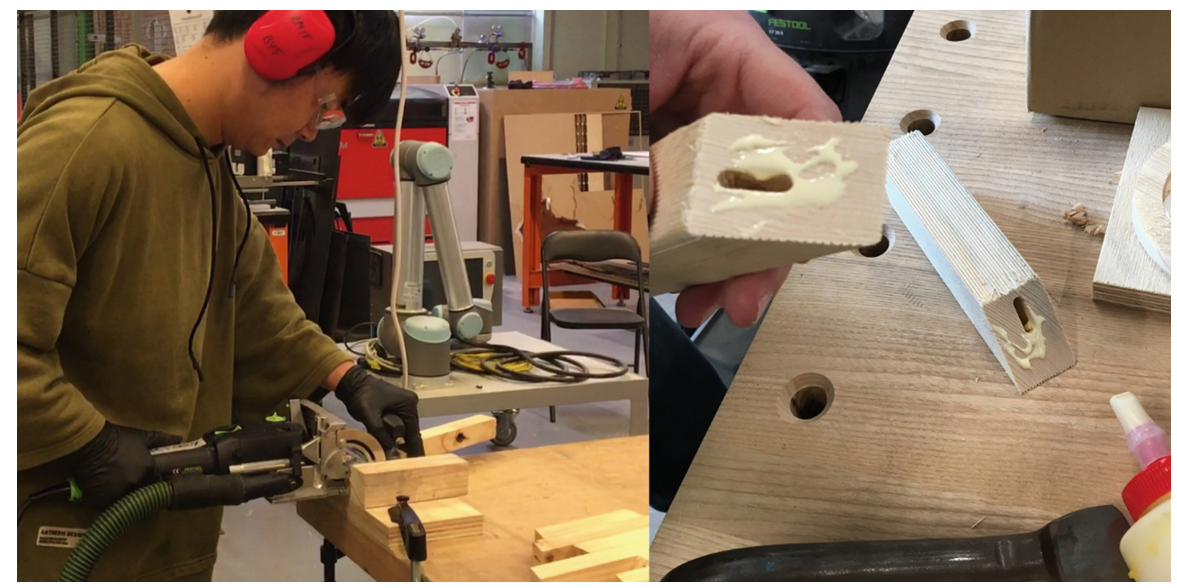

Fig. 5. A standardized timber domino system was used to fix the parts of the aggregation together

cutting process were used to stitch and brace these chunks together to enhance structural stability of the aggregation (Fig. 6).

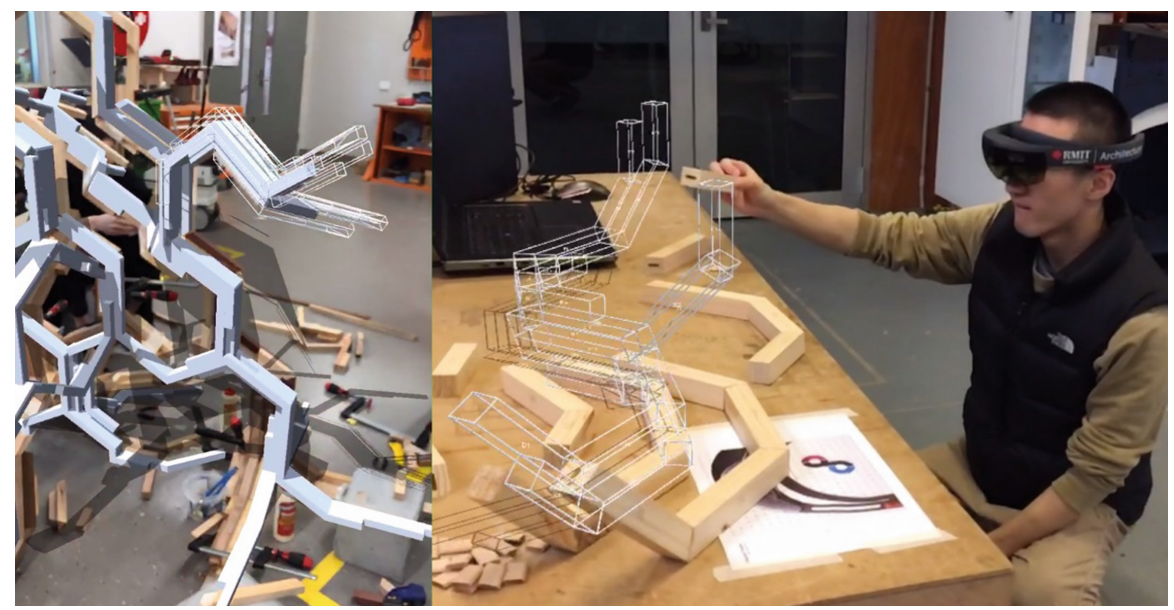

Fig. 6. The aggregation was assembles in chunks, and then together using a series of holographic guides

\subsection{Method 2 | Working to a Flexible Digital Model}

This second project focuses using non post processed recycled timber material and the development a flexible digital design model with the capacity to update in response to the digitised material inventory. While timber construction materials are to some degree standardised, one the challenges to using recycled materials is the variation in overall length and onsite modification made for during initial use phase. 


\subsubsection{The Digital Inventory}

High resolution 3D scanning is often used to create 'digital twins' of physical objects that can then be used and referenced within 3D models. These 3D scans however are complex setups (especially for part scanning), and result in workflows that require noise removal and digital post processing workflows to produce clean, accurate models (Stojanovic et al. 2018). Despite being odd shapes and sizes, each offcut timber scrap as rectangular in its shape, with a defined length, width and height. Rather than using 3D scanning (high digital cost) or measuring with a tape (high manual cost), Aruco markers were used in conjunction with Fologram's marker tracking tools to create digital twins of each of these offcut timber scraps. Participants would align a timber scrap at a holographic XY plane (set-out in physical space by a QR Origin code), and align an Aruco marker at the opposite corner diagonally, and tap once inside the HoloLens (see Fig. 7 and 8). This marker would send a location plane to Rhino \& Grasshopper and create a 2-point rectangular box of the dimensions of the timber scrap, thus creating an accurate digital twin of the offcut pieces with no post processing or manual measurement. The lowresolution digitised geometry becomes one of the library of parts that then informs the design.

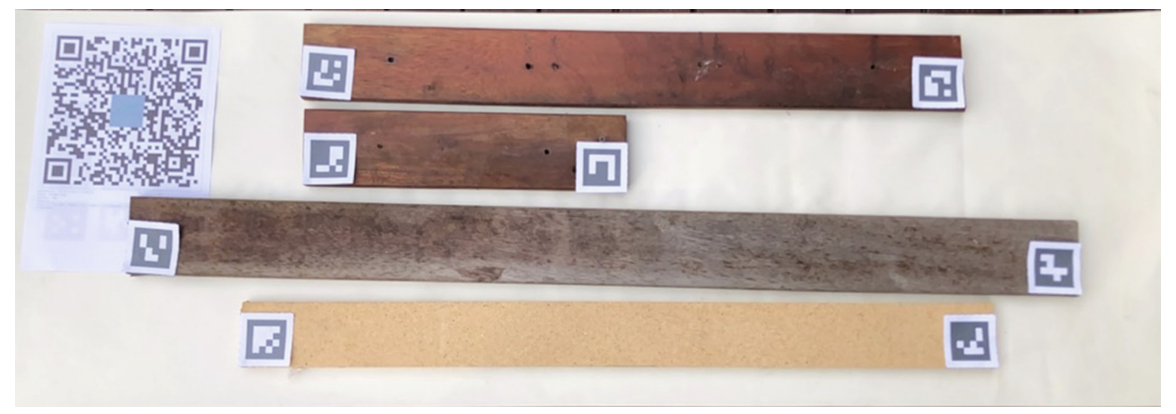

Fig. 7. Individual Aruco markers can be placed on top of each timber offcut and aligned to a $Q R$ Origin code

\subsubsection{Updating the Digital Design}

To begin the process, a base design is modelled which is updated and recreated live as each of the pieces are scanned. In this case, a simple undulating vertical wall was the design object and contoured into a series of curves that perform as guidelines which provide the preliminary alignment for the digital parts to align with. Each of the curves is assessed on its curvature and length against the inventory of digital lengths. Genetic solvers within Grasshopper (Mirjalili 2018) were used to establish a best fit of each of the timber lengths to sectional curves through the wall. This technique avoids having to cut or additionally process the timber lengths, further increasing its efficient reuse (Fig. 9).

The genetic solver then breaks down the contoured curves from the wall and tries to best fit the length of timbers. This capacity of the model to absorb differentially 

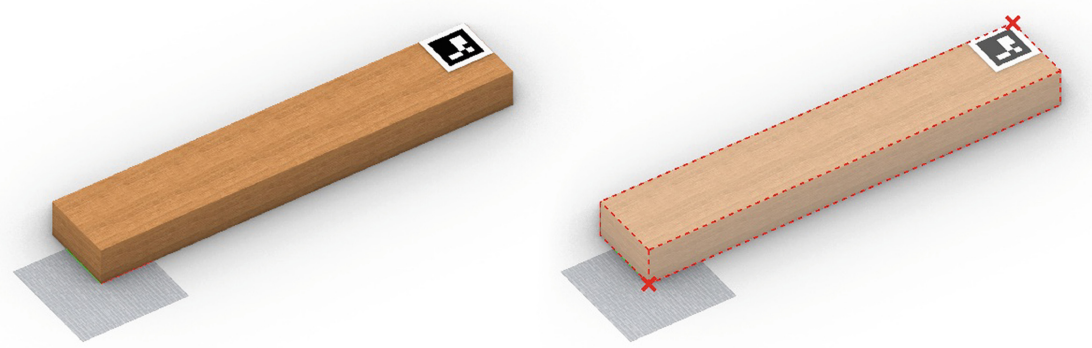

Fig. 8. Aruco marker tracking in Fologram \& a holographic origin point created 2-point boxes over the timber parts
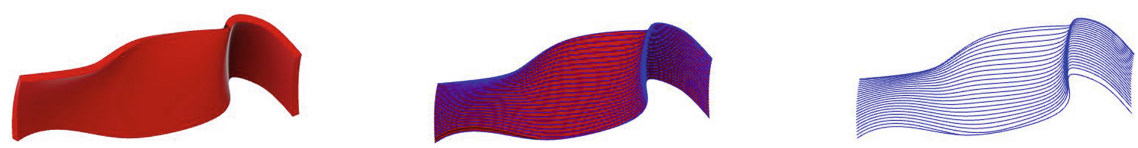

Fig. 9. Designed wall (left) contoured timber length guidelines (right)

dimensioned timber lengths encourages the emergence of highly textured 3D surfaces (Fig. 10).

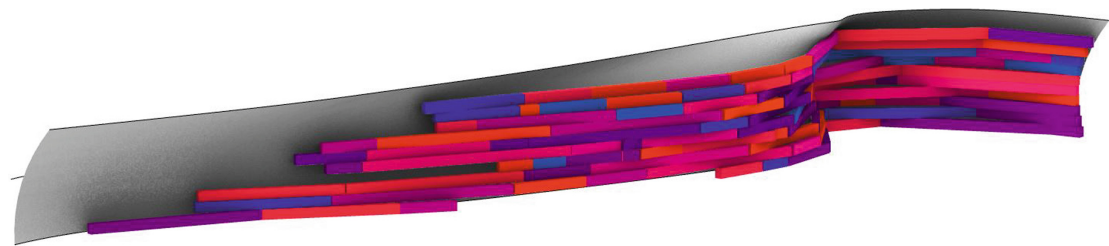

Fig. 10. Timber Lengths fitting to the original design

Some designs will be more efficient in how they use the timber lengths. As shown below in Fig. 8. The image on the left showing a shorted wall with greater curvature within the curve will not easily fit many of the pieces. In contrast, a longer less curvaceous wall will be more conducive with using more of the lengths of timber without the need for further cutting (Fig. 11).

\subsection{Results}

The method used for project 1 allowed for the use of uniquely shaped/mass customized timber pieces to assemble an aggregation made entirely of uniquely sized pieces. The nesting AR marking method reduced the wastage associated with each offcut timber piece. The end pieces meant participants could adjust their nesting dynamically to reduce offcut waste, or eliminate it entirely. Grouping the timber waste by cross section profiles 

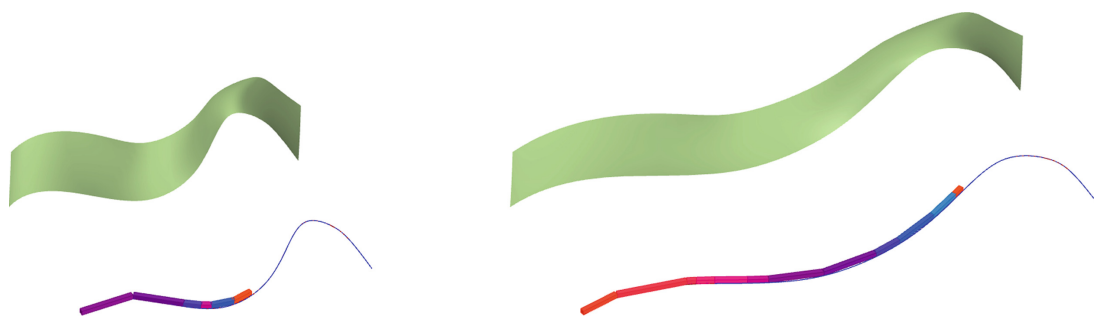

Fig. 11. Adjusting the digital model to increase efficient use of timber lengths

enabled the pieces to be located in the aggregation with a best fit method, which enabled the use of unique scrap material.

This method, however, came with complexities and problems. Many of the pieces did not have the required cross profile dimensions and were not used in the project. Some of the joints were poor and did not fit perfectly in the aggregation. The material still required machine manipulation through the cutting process needed to reach the aggregation piece lengths after marking, and still resulted in some (minor) wastage despite the nesting. This is compounded by the rigidness of the aggregation which did not respond to material input.

Project 2's method attempts to improve some of these processes of project 1 by creating an aggregation responsive to the material input. The digitization of each material scrap helped create a clear digital record of the pieces used in the aggregation

The capacity to update and experiment with the changing digital model meant that pieces could be used without any cutting or manipulation, which essentially removes all material wastage in the process and makes each scrap piece useable regardless of dimensions and cross sections.

\section{Discussion}

Providing a methodology to digitise and inventory land fill bound construction waste material has the potential to increase their reuse and recycling. Both of the projects discussed here demonstrate small non-structural objects designed and built from timber offcuts. The first project presents a more customised approach where augmented technology enables one to efficiently fit and cut the required parts to a static pre-designed object. The designed joint angles of the $\mathrm{L}$ system enabled nesting of the similar parts in an efficient manner and reduced the degree of cutting. This technique resulted in a monolithic well finished structure. In contrast to this, the second project had the capacity to absorb differentially sized and finished timber lengths creating a more textured outcome as the number of parts increase. The second project extended upon the first in establishing techniques for further reductions in material processing and inherent limitations of construction something predetermined from a randomised selection of timber length. The second project also provided the designer with a flexible relationship to the design and the digitised inventory of parts as opposed to the more traditional method of design then document. This method also has an underlying shift in the design paradigm 
where designers work from a blank slate of materials, where the only limiting factor is generally cost or structural competency.

\subsection{Future Development}

The process outlined in this paper offer two approaches to working with providing value to construction waste material. In the further evolution of this proof of concept it is intended to address more fully methods of geo-locating and stacking timber. Stacking the timber in such a way would take into account the order in which each piece is required in the construction process and then also guide the builder to the pieces whereabouts on site. Further work intends to establish methods to tag and link additional information to each scanned piece such as material type, structural/non-structural pieces or knots and blemishes in the material as a few examples. There is also more research to be done in creating a workflow that organizes the digital inventory and highlights the currents pieces location within the assembly in the augmented environment.

\section{References}

Jahn, G., Newnham, C., van den Berg, N., Iraheta, M., Wells, J.: Holographic construction. In: Gengnagel, C., Baverel, O., Burry, J., Ramsgaard Thomsen, M., Weinzierl, S. (eds.) DMSB 2019, pp. 314-324. Springer, Cham (2020)

Kaur, M.J., Mishra, V.P., Maheshwari, P.: The convergence of digital twin, IoT, and machine learning: transforming data into action. In: Farsi, M., Daneshkhah, A., Hosseinian-Far, A., Jahankhani, H. (eds.) Digital Twin Technologies and Smart Cities. IT, pp. 3-17. Springer, Cham (2020)

Krausmann, F., Wiedenhofer, D., Lauk, C., Haas, W., Hiroki, T., Fishman, T., Miato, A., Schandl, H., Haberl, H.: Global socioeconomic material stocks rise 23 fold over the 29th century and require half on annual resource use. In: Proceedings of the National Academy of Sciences, vol. 114, no. 8, pp. 1880 (2017)

Mirjalili, S.Z., Mirjalili, S., Saremi, S., Faris, H., Aljarah, I.: Grasshopper optimization algorithm for multi-objective optimization problems. Appl. Intell. 48(4), 805-820 (2017). https://doi.org/ 10.1007/s10489-017-1019-8

Mohammadi, N, Taylor, J.E.: Smart city digital twins. In: IEEE Symposium Series on Computational Intelligence (SSCI), Honolulu, HI, pp. 1-5 (2017). https://doi.org/10.1109/SSCI.2017. 8285439

Shooshtarian, S., Wong, S., Khalfan, M., Maqsood, T., Yang, J.: Green construction and construction and demolition waste management in Australia. In: Proceedings of the 43rd Annual Australasian University Building Educators Association Conference (AUBEA 2019), Noosa, Australia, 6-8 November 2019, pp. 18-25 (2019)

Stafford-Smith, M., Griggs, D., Gaffney, O., Ullah, F., Reyers, B., Kanie, N., Stigson, B., Shrivastava, P., Leach, M., O'Connell, D.: Integration: the key to implementing the sustainable development goals. Sustain. Sci. 12(6), 911-919 (2016). https://doi.org/10.1007/s11625-0160383-3 
Stojanovic, V., Trapp, M., Richter, R., Hagedorn, B., Döllner, J.: Towards the generation of digital twins for facility management based on 3D point clouds In: Gorse, C., Neilson, C. J. (eds.) Proceeding of the 34th Annual ARCOM Conference, 3-5 September 2018, Belfast, UK, pp. 270-279. Association of Researchers in Construction Management (2018)

Zou, P., Hardy, R., Yang, J.: Barriers to building and construction waste reduction, reuse and recycling: a case study of the Australian Capital Region. In: Panko, M., Kestle, L. (ed.) Proceedings of the Sustainability In Construction and Deconstruction Conference, Auckland, New Zealand, 15-17 July 2015, pp. 27-35

Open Access This chapter is licensed under the terms of the Creative Commons Attribution 4.0 International License (http://creativecommons.org/licenses/by/4.0/), which permits use, sharing, adaptation, distribution and reproduction in any medium or format, as long as you give appropriate credit to the original author(s) and the source, provide a link to the Creative Commons license and indicate if changes were made.

The images or other third party material in this chapter are included in the chapter's Creative Commons license, unless indicated otherwise in a credit line to the material. If material is not included in the chapter's Creative Commons license and your intended use is not permitted by statutory regulation or exceeds the permitted use, you will need to obtain permission directly from the copyright holder.

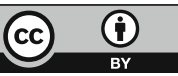

